\title{
In Memoriam Helmut Koch
}

\author{
Winfried Mellwig
}

Online publiziert: 31. März 2016

(C) Schmalenbach-Gesellschaft für Betriebswirtschaft e.V. 2016

Am 7. August 2015 verstarb kurz vor Vollendung seines 96. Lebensjahres Prof. Dr. Dr. h.c. Helmut Koch, emeritierter Professor für Betriebswirtschaftslehre an der Universität Münster. Mit ihm verliert die deutsche Betriebswirtschaftslehre einen ihrer profiliertesten Vertreter aus der Hochschullehrergeneration, die nach dem 2. Weltkrieg die theoretische Neuorientierung des Faches begründete.

Helmut Koch wurde am 24. September 1919 in Oberlübbe, Kreis Minden/ Westfalen, als Pfarrerssohn geboren. Nach einer Kriegsverwundung studierte er von 1941 bis 1945 an den Technischen Hochschulen Hannover und Berlin-Charlottenburg; er legte 1945 die Diplomprüfung im Fach Wirtschafts-Ingenieurwesen ab. Von 1945 bis 1949 war er Mitarbeiter von Karl-Wilhelm Hennig an der TH Hannover und wurde dort 1948 mit der Arbeit „Die industrielle Kalkulation als Stufenrechnung“ zum Dr. Ing. promoviert. Er studierte sodann Betriebswirtschaftslehre und ging 1949 als wissenschaftlicher Assistent zu Erich Gutenberg an die Universität Frankfurt am Main, wo er 1950 die Diplom-Prüfung für Kaufleute ablegte. Nach seiner Habilitation 1951 mit einer unveröffentlicht gebliebenen Arbeit zur Betriebsgröße (,Die optimale Produktmenge als Gegenstand langfristiger Planung“) lehrte er zunächst als Privatdozent. Nach Ablehnung einiger Auswärtsrufe wurde er 1955 zum persönlichen Ordinarius ernannt und übernahm den Lehrstuhl für Revisionsund Treuhandwesen an der Frankfurter Wirtschafts- und Sozialwissenschaftlichen Fakultät.

1957 folgte Helmut Koch einem Ruf auf einen Lehrstuhl an der Rechts- und Staatswissenschaftlichen Fakultät der Universität Münster als Direktor des Instituts für Betriebsanalysen (später Institut für Industrielle Unternehmensplanung). Trotz

\footnotetext{
W. Mellwig $(\bowtie)$

Goethe-Universität,

Frankfurt am Main, Deutschland

E-Mail: w.mellwig@t-online.de
} 
zahlreicher attraktiver Rufe an andere Universitäten (Bonn, Göttingen, Mannheim, München, Saarbrücken) ist der Westfale der Universität Münster bis zu seiner Emeritierung 1984 treu geblieben.

Die Arbeiten Kochs belegen sein weitgespanntes wissenschaftliches Interesse und zeugen von seiner Fähigkeit, Fachprobleme weitab von eingetretenen Pfaden zu diskutieren. Seine Publikationen umfassen, bei grober Einteilung, folgende Gebiete: methodologische und konzeptionelle Grundfragen der Betriebswirtschaftslehre, Ungewissheitstheorie, Bilanzierung, Produktions- und Kostentheorie, Kostenrechnung und kurzfristige Erfolgsrechnung, Unternehmensplanung.

Von der Fähigkeit Kochs, scheinbar Bewährtes in Zweifel zu ziehen, zeugt zunächst eine Arbeit aus seiner Zeit als Ordinarius für Treuhandwesen in Frankfurt. Der Beitrag „Die Problematik des Niederstwertprinzips“, 1957 in „Die Wirtschaftsprüfung" erschienen, wurde aufgrund der Neuartigkeit der Gedanken heftig kritisiert. In einer Zeit, da man den niedrigeren Alternativwert zu den Anschaffungs- und Herstellungskosten einhellig an Wiederbeschaffungspreisen orientierte, musste wohl die Ableitung des Alternativwertes aus der Entwicklung am Absatzmarkt auf Widerstand stoßen. Das Niederstwertprinzip als gesetzliche Kodifizierung des Imparitätsprinzips („Grundsatz der Verlustantizipation“) kann in der Tat keine Antizipation von Gewinnminderungen, sondern lediglich die Berücksichtigung von künftigen Verlusten, sprich: die Orientierung am Absatzmarkt, begründen. Die Ablehnung der Gedanken Kochs wich allerdings alsbald breiter Zustimmung in Theorie und Praxis. In seinem Aufsatz „Zur Problematik des Teilwertes“ (ZfhF 1960) übertrug er seine Gedanken auf die steuerliche Bilanzierung.

Im Bereich der Unternehmensrechnung hat sich Helmut Koch ferner intensiv mit Grundfragen der Kostenrechnung und der kurzfristigen Erfolgsrechnung befasst. Breit diskutiert wurden in der Literatur vor allem seine Gedanken zum Kostenbegriff (ZfhF 1958, ZfB 1959) - Koch war der wohl profilierteste Vertreter des sog. pagatorischen Kostenbegriffs - und zur Gemeinkostenschlüsselung (ZfbF, ZfB 1965). Drei dieser Aufsätze wurden, durch einen weiteren Beitrag ergänzt, 1966 in dem Buch „Grundprobleme der Kostenrechnung“ veröffentlicht, dessen mehrfache Neudrucke den Widerhall auch dieser Arbeiten dokumentieren.

Die besonderen wissenschaftlichen Neigungen Kochs aber lagen auf dem Gebiete der Unternehmenstheorie. Sein erster Aufsatz (Zeitschrift für die gesamte Staatswissenschaft 1950) befasste sich, wohl maßgeblich motiviert durch seinen Lehrer Erich Gutenberg, mit Überlegungen zum Gültigkeitsbereich des Ertragsgesetzes. Seine eigene Auffassung zu diesem Problemfeld hat er 1966 in einem Beitrag zur Festschrift für Karl Hax verdeutlicht.

Wesentliches Element der Arbeiten Kochs waren auch Probleme der Entscheidungsfindung bei Ungewissheit. Seine ,Theorie der Sekundäranpassung“ (erste Veröffentlichung ZfhF 1960), später zur „Theorie des Gewinn-Vorbehalts“ weiterentwickelt, beruht letztlich auf einem tiefen Zweifel an der Gültigkeit des Substitutionsoder Stetigkeitsaxioms der Risikonutzentheorie, an der uneingeschränkten Risiko/ Chancen-Substitition. Die Kochsche Konzeption zur Ungewissheitstheorie kann insofern als eine Alternative zum Bernoulli-Prinzip verstanden werden.

Den zentralen Schwerpunkt der theoretischen Arbeiten Kochs bilden konzeptionelle Fragen der betriebswirtschaftlichen Theorie. Seit seinem vielbeachteten Auf- 
satz „Über einige Grundfragen der Betriebswirtschaftslehre“ (ZfhF 1957) haben ihn die Grundlagen der betriebswirtschaftlichen Theorie nicht mehr losgelassen. Eine erste geschlossene Darstellung stellt 1962 sein Beitrag „Über eine allgemeine Theorie des Handelns" in der ersten der drei von ihm herausgegebenen Gutenberg-Festschriften dar. Die Handlungstheorie, sprachlich wohl präziser: Theorie des Handelns, wurde in den Folgejahren stetig ausgebaut. Hiervon zeugen zahlreiche Aufsätze, unter denen der wichtigste sicherlich der Beitrag „Die Theorie der Unternehmung als Globalanalyse“ (Zeitschrift für die gesamte Staatswissenschaft 1964 ) ist. Dieser nimmt Gedanken vorweg, auf die sich die Betriebswirtschaftslehre erst gut ein Jahrzehnt später besinnt, nachdem die euphorische Phase der Simultanplanung einer gewissen Ernüchterung gewichen war. Einen Höhepunkt, wenngleich keinen Abschluss, haben die einschlägigen Arbeiten Kochs in dem 1975 erschienen Buch „Die Betriebswirtschaftslehre als Wissenschaft vom Handeln, Die handlungstheoretische Konzeption der mikroökonomischen Analyse" gefunden, ein Werk, das in dieser Zeitschrift 1977 von Waldemar Wittmann ausführlich gewürdigt wurde. Die handlungstheoretische Konzeption der Betriebswirtschaftslehre ist Ergebnis jahrzehntelangen Ringens um wissenschaftlichen Fortschritt, getragen von einem leidenschaftlichen Arbeitswillen, der seine Mitarbeiter, die in einer Vielzahl von Diskussionen an der Entwicklung der Gedanken teilhaben konnten, immer wieder aufs Neue faszinierte.

Helmut Koch versteht die Handlungstheorie als eine Basisdisziplin, die eine Formulierung fachspezifischer Besonderheiten in Forschungsgegenstand und Forschungsmethode gestattet, die trotz der Notwendigkeit zur Spezialisierung in Einzeldisziplinen den Zusammenhalt des Faches zu sichern erlaubt. Die Geschlossenheit der wirtschaftswissenschaftlichen Forschung war ihm zentrales Anliegen. Dass sich Helmut Koch nicht als Betriebswirt, sondern im weiteren Sinne als Wirtschaftswissenschaftler verstand, geht sicherlich in hohem Maße auch auf Impulse zurück, die er während seiner Zeit in Hannover durch den von ihm sehr verehrten Wilhelm Kromphardt empfangen hat. Der unbeirrte Einsatz Kochs gegen eine Trennung von Volkswirtschaftslehre und Betriebswirtschaftslehre (,Von seiner Mutter trennt man sich nicht.") und für ein Zusammengehen in einer Fakultät ist vor diesem Hintergrund nur folgerichtig. Dem trug auch der Titel der zweiten Festschrift für Helmut Koch 1989 („Der Integrationsgedanke in der Betriebswirtschaftslehre“) Rechnung. Kochs Bemühen, isolierte Gebiete betriebswirtschaftlicher Forschung zu einer geschlossenen Theorie zusammenzuführen, wurde 1981 an der Universität Hamburg mit der Verleihung der Ehrendoktorwürde des Fachbereichs Wirtschaftswissenschaften gewürdigt.

Schließlich ging es Helmut Koch um eine möglichst enge Verklammerung theoretischer Erkenntnisse mit der Praxis der Unternehmensführung. Die betriebswirtschaftliche Theorie als erste Stufe der Erkenntnisgewinnung stellt bei Koch konsequent auf das unternehmerische Handeln und seine Determinanten ab; sie ist insofern einzelwirtschaftlich (,individualanalytisch“) konzipiert und wird deutlich abgegrenzt von der überkommenen neoklassischen Konzeption gesamtwirtschaftlicher (,,marktorientierter") Gleichgewichtsanalyse. In einer zweiten Stufe steht das Bemühen, die gewonnenen theoretischen Erkenntnisse der Entscheidungsfindung in Unternehmen nutzbar zu machen, d.h. praktikable Entscheidungskriterien zu gewinnen. Dieser 
Anwendungsbezug der Betriebswirtschaftslehre trat zunehmend in den Vordergrund. Die zahlreichen Arbeiten Kochs zur Unternehmensplanung sind nichts anderes als eine Übertragung des theoretischen Gedankengebäudes auf die Bedingungen der Unternehmenspraxis, die er durch seine engen Kontakte zur Unternehmenspraxis zu durchleuchten bemüht war. Seine Publikationen zur Unternehmensplanung, beginnend mit der Monographie „Betriebliche Planung, Grundlagen und Grundfragen der Unternehmungspolitik“ von 1961 bis zu den späteren Untersuchungen „Aufbau der Unternehmensplanung“ 1977, „Neuere Beiträge zur Unternehmensplanung“ 1980 und „Integrierte Unternehmensplanung“ 1982, wurden zu Standardliteratur auf diesem Gebiet. Die Verbindung von theoretischer Analyse und Unternehmenspraxis kennzeichneten auch die Vorlesungen und Seminare Kochs. Zahlreiche Chefs großer Unternehmen wurden zu Vortrag und Diskussion nach Münster geholt.

Diese Grundanliegen Kochs fanden nicht zuletzt ihren Niederschlag in seinen Aktivitäten in wissenschaftlichen Gesellschaften. Von 1962 bis 1966 war er stellvertretender Vorsitzender im Verein für Socialpolitik, danach Mitglied des erweiterten Vorstands. Die Gründung der Ausschüsse für Unternehmenstheorie und -politik sowie für Unternehmensrechnung geht maßgeblich auf die Initiativen Kochs zurück. In der ,alten“ Schmalenbach-Gesellschaft war Koch lange Jahre Mitglied des Verwaltungsrates und nach der Verschmelzung mit der Deutschen Gesellschaft für Betriebswirtschaft zur ,neuen“ Schmalenbach-Gesellschaft gehörte er dort dem Vorstand an. Ferner war Koch Mitglied des Kuratoriums des Universitätsseminars der Wirtschaft (USW), der zentralen Ausbildungsstätte für Führungskräfte der Wirtschaft.

Helmut Koch war ein Mann mit ausgeprägten musischen Neigungen. Da ist zum einen seine Liebe zur Musik. Bis in die späten Jahre waren ihm Reisen mit auch weiten Wegen zu verschiedenen Opernhäusern nicht zu beschwerlich. Seine große Leidenschaft galt der Querflöte und dabei der Musik des Barock. Bis ins Alter hinein übte er täglich mit und ohne Lehrer.

$\mathrm{Da}$ ist zum anderen seine Begeisterung für die Malerei der Moderne zu nennen. Mit großer Sachkenntnis konnte er dem Zuhörer die Malerei von Fernand Léger, Serge Poliakoff, Karl Schmidt-Rottluff und anderen nahebringen. Insbesondere Ernst Wilhelm Nay und Fritz Winter hatten es ihm angetan. Bei Nay schätzte er vornehmlich die in Öl gearbeiteten „Scheibenbilder“, die wuchtig konzipiert den Betrachter aufwühlen, aber auch kleinere Arbeiten, Gouachen, mit herrlichen Gelb-, Blau- und Rottönen. Seine Vorliebe für Fritz Winter als Vertreter expressionistischer Abstraktion galt vor allem dem in Öl gemalten Farbfelderzyklus, aber auch kleineren Farblithographien, die in ihrer planvollen Abstraktion der Fantasie des Betrachters freien Lauf lassen.

Für seine Schüler war Helmut Koch ein verständnisvoller und einfühlsamer Lehrer. Er konnte zuhören und, wenngleich in seinem wissenschaftlichen Standort gefestigt, war er Argumenten anderer jederzeit zugänglich. Er ließ seinen Schülern den Freiraum und vor allem die Muße, die sie für ihre eigene fachliche Orientierung und Entwicklung benötigten. Dies haben seine Mitarbeiter stets als besondere Großzügigkeit geschätzt. Wir werden diesen großartigen Menschen und väterlichen Freund sehr vermissen. 